\title{
Geomagnetic jerks from the Earth's surface to the top of the core
}

\author{
Aude Chambodut ${ }^{1,2 *}$, Céline Eymin ${ }^{3}$, and Mioara Mandea ${ }^{2}$ \\ ${ }^{1}$ Department of Applied and Industrial Mathematics, University of Potsdam, Postfach 601553, D-14415 Potsdam, Germany \\ ${ }^{2}$ GeoForschungsZentrum Potsdam, Telegrafenberg, D-14473 Potsdam, Germany \\ ${ }^{3}$ Laboratoire de Géophysique Interne et Tectonophysique, Université Joseph Fourier, BP53, 38041 Grenoble Cedex 9, France
}

(Received October 6, 2006; Revised June 1, 2007; Accepted June 6, 2007; Online published July 20, 2007)

\begin{abstract}
Rapid changes in the magnetic field characterised by an abrupt change in the secular variation have been named "secular variation impulses" or "geomagnetic jerks". Three of these events, around 1968, 1978 and 1990 , occurred during the time-span covered by the comprehensive model CM4 (Sabaka et al., 2002, 2004). This model, providing the best temporal resolution between 1960 and 2002 as well as a fine separation of the different magnetic sources, can be used to study rapid phenomena of internal origin. In order to analyse these events all over the globe, synthetic time series were obtained from the CM4 model between 1960-2002. Geomagnetic jerks are detected here as a rapid movement of the zero isoline of the second field derivative. Analysis of the area swept out by this isoline as a function of time allows us to map the spatial extent of jerks though time, and to identify an event around 1985 that is localized in the Pacific area. At the core surface, we compute the fluid flows under the frozen-flux and tangentially geostrophic assumptions. The flows do not exhibit any special pattern at jerk times, but instead show a smooth temporal evolution over the whole time period. However, the mean amplitude of the dynamical pressure associated with these flows present maxima at each jerk occurrence and helps to confirm the identification of a jerk in 1985 .
\end{abstract}

Key words: Geomagnetic jerks, magnetic models, core surface flows, core dynamics.

\section{Introduction}

The Earth's magnetic field occasionally shows rapid changes in its temporal variations. These events are known as "secular variation impulses" or "geomagnetic jerks".

The extent, duration and the underlying processes causing geomagnetic jerks are still debated. Indeed, the question of whether geomagnetic jerks are external or internal in origin has been a matter of debate since the 1980 s, mainly because of the difficulty in separating internal from external contributions to the magnetic data. Some authors suggested an external origin (e.g. Alldredge, 1984, 1985), as the rapid geomagnetic secular acceleration could be interpreted as a part of the well-known solar cycle effect. An internal origin has been supported by a variety of analyses and authors (e.g. Malin and Hodder, 1982; Courtillot et al., 1984; Malin et al., 1983; Courtillot and Le Mouël, 1985; McLeod, 1985; Gavoret et al., 1986; Backus et al., 1987; Golovkov et al., 1989; Alexandrescu et al., 1995, 1996; Bellanger et al., 2001; Bloxham et al., 2002). Although an internal origin is now widely accepted, the processes causing geomagnetic jerks as a whole are not yet wellunderstood.

At the Earth's surface, geomagnetic jerks in the field components are represented as two second-degree polynomials of time with a change in curvature at the times of

*Now at Department of Magnetic Observatories, École et Observatoire des Sciences de la Terre, 67084 Strasbourg Cedex, France.

Copyright (c) The Society of Geomagnetism and Earth, Planetary and Space Sciences (SGEPSS); The Seismological Society of Japan; The Volcanological Society of Japan; The Geodetic Society of Japan; The Japanese Society for Planetary Sciences; TERRAPUB the event; the corresponding secular variation (the first time derivative of the geomagnetic field) has a V-shaped feature, the second time derivative is step like and the third derivative is like a Dirac-delta function (Courtillot et al., 1984). However, this definition of a geomagnetic jerk observed at one point at the Earth's surface is an ideal one.

Some previous studies have already attempted to characterise the temporal occurrences and extents of geomagnetic jerks at the Earth's surface using the magnetic field itself (Alexandrescu et al.,1996; Nagao et al., 2002) or its secular variation (Macmillan, 1996; Le Huy et al., 1998; De Michelis et al., 1998; Chambodut and Mandea, 2005). Alexandrescu et al. (1996) note the non-synchronous occurrence of geomagnetic jerks at global scale: a time shift appears for the events around 1968 and 1978 with an earlier arrival in the Northern hemisphere. Le Huy et al. (1998) find an "anti-correlated" character in successive secular variation impulses. The events around 1968 and 1990 appear similar in their shape and sign while the one of 1978 shows a change of sign. More recently, Chambodut and Mandea (2005) used the CM4 model (Sabaka et al., 2002, 2004) to study geomagnetic jerks, and suggested that these events might not be worldwide in occurrence.

At the core surface, flow motions can be used to investigate geomagnetic jerks. Because of the high electrical conductivity of the fluid in the outer core, advection of the magnetic field by the flow dominates over diffusion. This "frozen flux" approximation (Roberts and Scott, 1965) has been used previously to construct global maps of the large-scale core flow. The determination of core flow is nonetheless highly non-unique, and more assump- 
tions have to be made in order to reduce or resolve this non-uniqueness. Different assumptions have been used so far to reduce this non-uniqueness: the flow is constrained to be purely toroidal (Whaler, 1980; Bloxham, 1990), to be tangentially geostrophic (Hills, 1979; Le Mouël et al., 1985; Gire et al., 1986; Backus and Le Mouël, 1986, 1987) or to be steady over a definite time span (Gubbins, 1982; Voorhies, 1984, 1986; Voorhies and Backus, 1985).

Some previous studies have already attempted to characterise the behaviour of the core surface flows during a geomagnetic jerk. However, not all kinds of flows are compatible with these rapid modifications of the magnetic field-a stationary flow being an example (Gubbins, 1984; Davis and Whaler, 1993; Voorhies, 1995; Bloxham et al., 2002). Waddington et al. (1995) and Davis and Whaler (1997) investigated instantaneous changes in flows and the occurrence of geomagnetic jerks at the Earth's surface. Hulot et al. (1993) identified a temporal change in the slope of the flow energy at jerk times. Le Huy et al. (2000) observed similarities in the pattern of the acceleration of the very large-scale flows during different geomagnetic jerks. More recently, Bloxham et al. (2002) showed that a timedependent flow may, at a given moment and location, produce a jerk signal, because of a particular geometry of the magnetic field rather than because of a particular dynamical event.

Because of the sparse distribution of geomagnetic observatories, the first source of data for direct analysis of geomagnetic jerks, we turn here to a global description of the magnetic field, through available models. In this study, we use the comprehensive geomagnetic field model CM4 (Sabaka et al., 2002, 2004). At first, we use this model as a tool to study the spatial occurrence of geomagnetic jerks at the Earth's surface. We analyse in detail the behaviour of the second time derivative, obtained from the spherical harmonic model of Sabaka et al., only for the internal field (i.e. up to degree/order 13) over the 40-year period of the model. We then concentrate on the core surface, using the comprehensive model CM4 to analyse, more accurately than was previously possible, the modifications of core surface flows linked to the jerks.

\section{Distribution of Geomagnetic Jerks Inferred from CM4 Secular Acceleration}

The secular variation of the magnetic field from observatory data was already compared with that from the comprehensive model CM4 by Chambodut and Mandea (2005), where a good agreement between the real and synthetic data was shown. The secular acceleration calculated from the CM4 model should also be a possible tool to detect the signature of geomagnetic jerks as sudden changes of sign of the secular acceleration (Courtillot et al., 1978). Searching for geomagnetic jerks in the second field derivative has not been attempted, mainly because of the noise level in such series computed from real observatory data. Using synthetic main-field data, provided by the CM4 model, the noise contribution is minimised as the model already takes into account the external fields and smooths out residual noise fluctuations.

\subsection{At observatories}

We observe the signature of geomagnetic jerks in the secular acceleration computed from the CM4 model at 39 observatory locations, which provide continuous monthlymean series during the period 1960-2002 (the same set of observatories as in Chambodut and Mandea (2005) is used here). As an example, Fig. 1 shows the second time derivative of each of the magnetic field components computed from the CM4 model at two observatory locations: Chambon la Forêt (CLF) situated in Europe $\left(48.023^{\circ} \mathrm{N}\right.$; $\left.2.260^{\circ} \mathrm{E}\right)$ and Apia (API) located in the Pacific $\left(13.807^{\circ} \mathrm{S}\right.$; $\left.171.775^{\circ} \mathrm{W}\right)$.

For the CLF observatory, the $\ddot{X}$ (North magnetic secular acceleration) and $\ddot{Z}$ (down vertical magnetic secular acceleration) components show oscillatory behaviour, with many zero level crossings. This makes it difficult to precisely indicate the occurrence of a jerk. The $\ddot{Y}$ (East magnetic secular acceleration) curve crosses the zero line four times, in 1969.5, 1981.7, 1991.2 and 1997.0 (two last intersection points after 2000 are not considered here, both of which are outside the interval studied). The first and third noted events, (around 1970 and 1990, respectively) are particularly well defined. These dates are confirmed by previous studies and analyses of time series at this observatory (see for instance Alexandrescu et al., 1996). The second event is dated with an uncertainty of \pm 1 year, as the change of sign appears after a small inflection in the $\ddot{Y}$ curve.

For the API observatory, the $\ddot{Y}$ curve shows two welldefined jerks around 1970 and 1978. The situation is however more complicated than for CLF observatory secular acceleration, as many crossing points of the zero line appear. A couple of oscillations could be due to some remaining external noise effects (mainly over the first decade). Some $\ddot{Y}$ values are very close to zero over a couple of years around 1986.

These examples underline the complexity of the acceleration field behaviour. Even with synthetic data-a smoothed version of reality - the geomagnetic jerks are sometimes difficult to detect.

\subsection{Over the whole Earth}

Since the secular acceleration can be used to localise geomagnetic jerks, we choose to study the positions of the zero isoline of the secular acceleration over the whole sphere. $\ddot{X}$, $\ddot{Y}$ and $\ddot{Z}$ values are computed only for the core field contribution (i.e. up to spherical harmonic degree/order 13) from the CM4 model.

To find the positions where the second derivative of the magnetic field is vanishing, indeed the surface swept by the zero isoline, the following algorithm is applied with a timespan of 0.1 year:

$$
\left\{\begin{array}{l}
\text { if }\left(\ddot{E}_{k}\left(t_{1}\right) \times \ddot{E}_{k}\left(t_{2}\right)\right)<0 \\
\rightarrow \ddot{E} \text { changes sign between } t_{1} \text { and } t_{2} . \\
\text { if }\left(\ddot{E}_{k}\left(t_{1}\right) \times \ddot{E}_{k}\left(t_{2}\right)\right) \geq 0 \\
\rightarrow \ddot{E} \text { does not change sign between } t_{1} \text { and } t_{2} \text {. }
\end{array}\right.
$$

where $\ddot{E}$ denotes one of the component of the acceleration vector field (e.g. $\ddot{X}, \ddot{Y}$ or $\ddot{Z}$ ).

In order to get a global description, we compute the surface integral $S$ on the sphere $\Omega$ covered by the areas satisfy- 

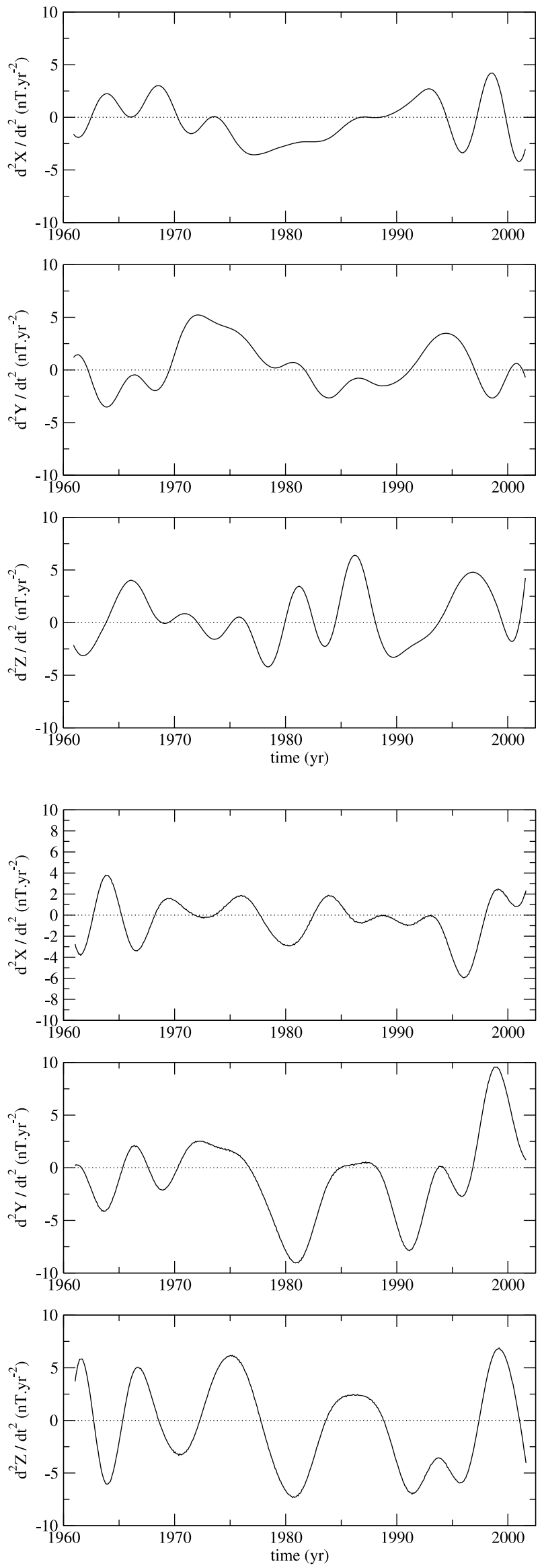

Fig. 1. Secular acceleration, $\ddot{X}, \ddot{Y}$ and $\ddot{Z}$ components, at CLF (Chambon la Forêt, France) observatory (top) and API (Apia, Western Samoa) observatory (bottom) computed from the CM4 model.
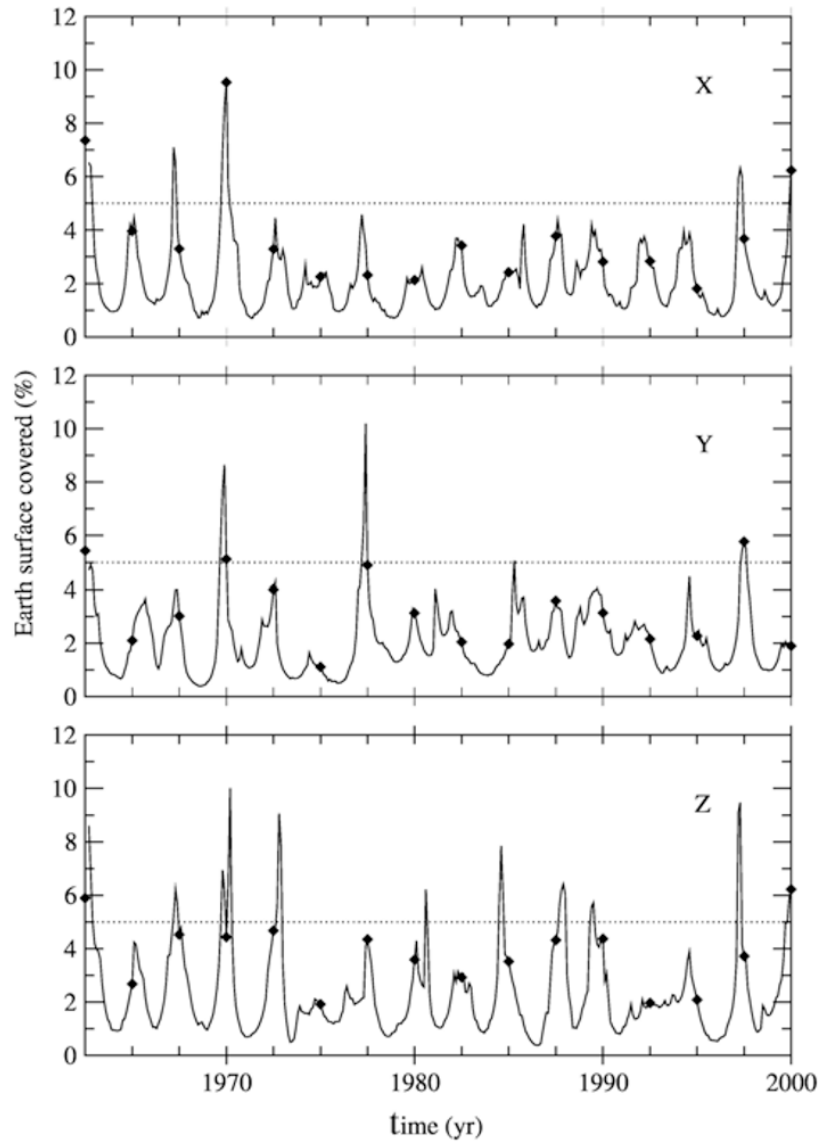

Fig. 2. Function $S(t)$ : Percentage of the Earth's surface covered by the movements of the zero isoline of $\ddot{E}$. The black diamonds represent the time of cubic B-splines knot spacing used in the CM4 model.

ing condition (1) for each magnetic component of the field and for each epoch $t$ :

$$
S(t)=\frac{1}{4 \pi} \int_{\Omega} \delta(t) d s
$$

where: $\left\{\begin{array}{l}\delta(t)=1, \text { if case (1) is satisfied at } t=\frac{t_{1}+t_{2}}{2} \\ \delta(t)=0, \text { if case (2) is satisfied at } t=\frac{t_{1}+t_{2}}{2}\end{array}\right.$.

The values of the $S(t)$ function reveal the extent of zones covered by the isoline of $\ddot{E}$. The maxima of this function denote large areas covered, in a short period of time, by the zero isoline of $\ddot{E}$ at the Earth's surface.

The graphs of $S(t)$ are shown in Fig. 2. The first observed feature is a 2.5 -year periodic signal (represented as black diamonds on curves of Fig. 2). That variation is not surprising as the CM4's secular variation is modeled through cubic Bsplines with a time knot spacing of 2.5 years. However, the influence of the cubic B-splines does not explain all variations of the shown curves ; indeed, several maxima occur far enough from any knot to suggest they have no link to the cubic-B-spline basis. We estimate a value: $S(t)=5 \%$ arises mainly due to cubic B-splines. We determine this threshold by taking into account the noise level of $S(t)$ produced by a time-dependant magnetic field model over 40 years with arbitrary linear secular variation and temporal cubic B-splines knot spacing of 2.5 years. Explicitly, the 

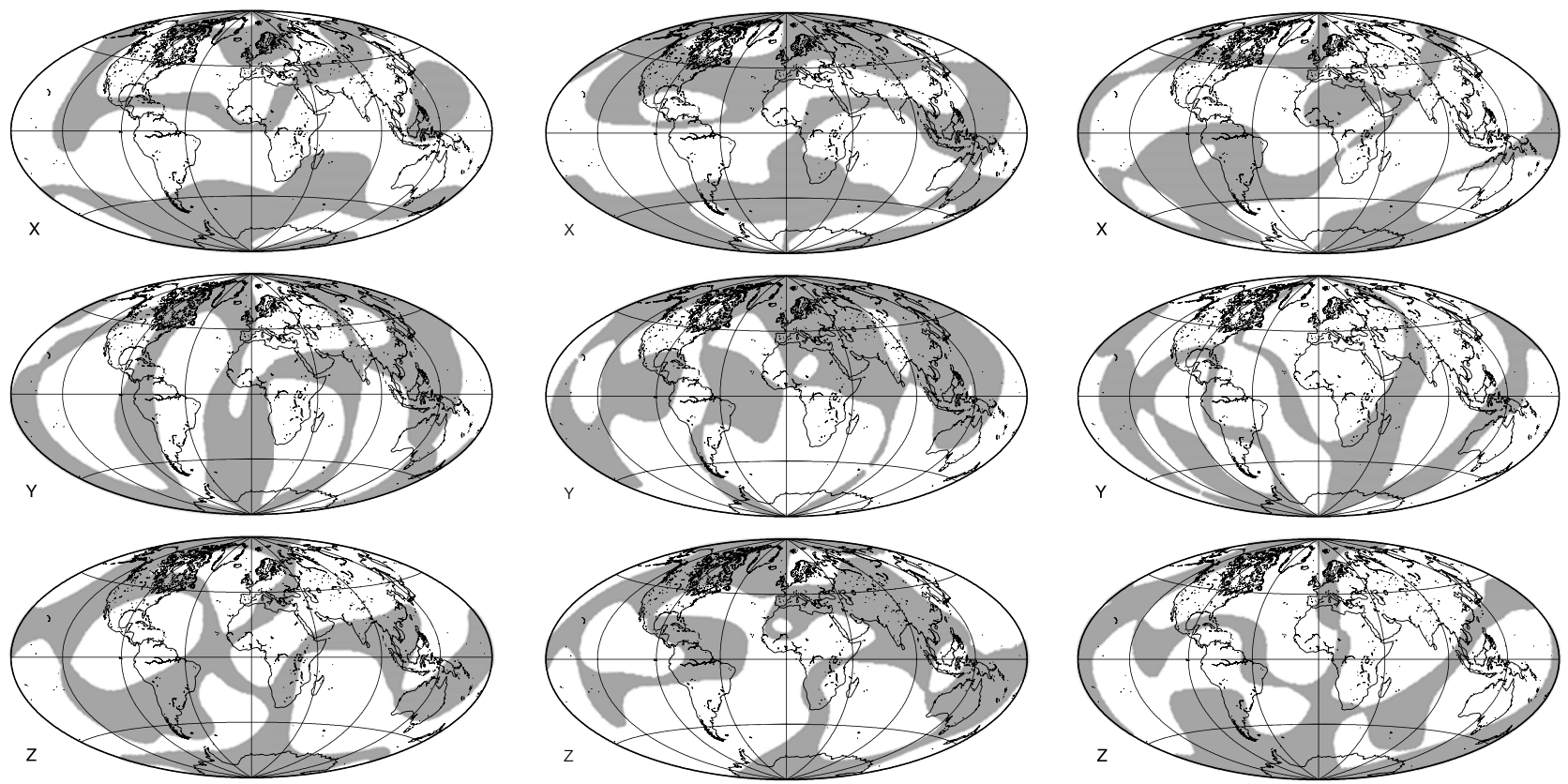

Fig. 3. Maps indicating locations where the zero isoline "swept" the Earth's surface, so where the geomagnetic jerk appears. From left to right, the three panels correspond to three one-year periods centred onto: $1967.2,1970.0$ and 1972.7, epochs corresponding to the maxima $S(t) \geq 5 \%$.
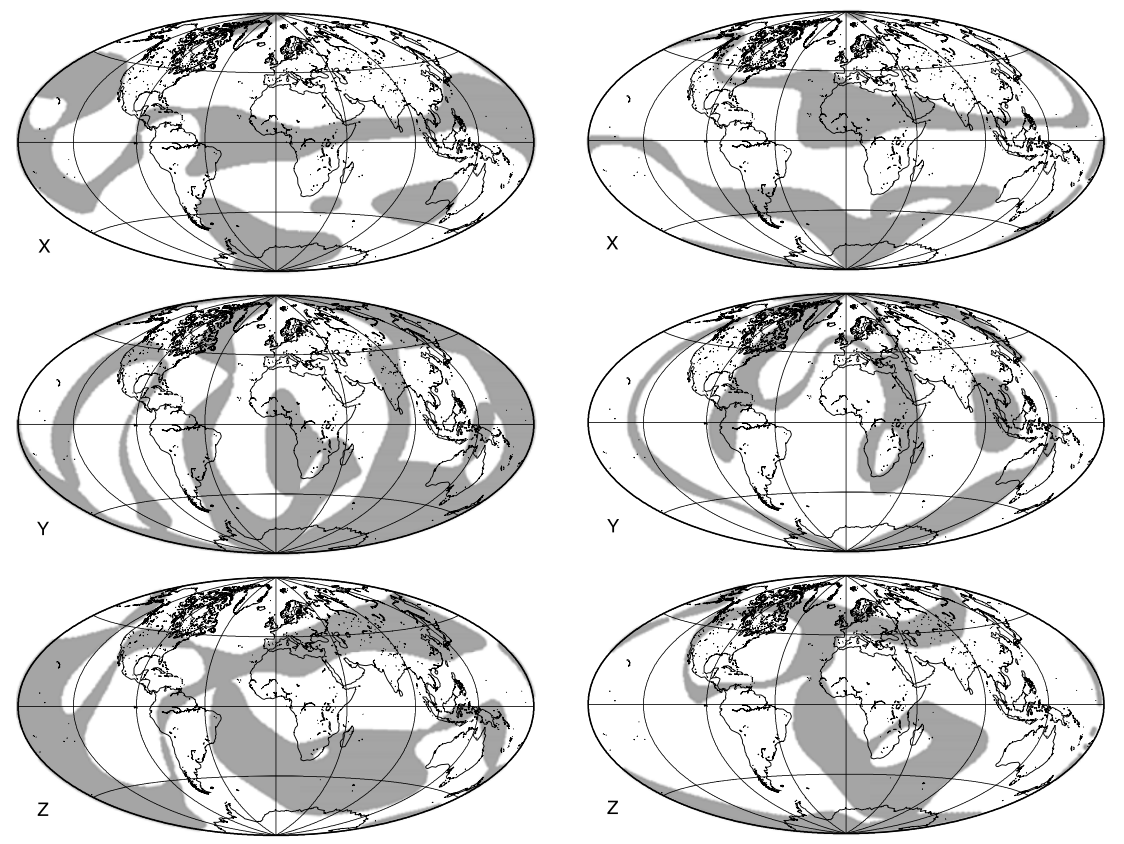

Fig. 4. Same caption as Fig. 3 but for two one-year periods centred onto: 1977.5 (left) and 1980.5 (right).

boundary value of $5 \%$ is larger than the fraction of Earth's surface covered by Europe. In the following, we discuss these maxima which are not linked to artifacts introduced by the modelling approach:

(i) For the $\ddot{X}$ component curve, three maxima can be noted in 1967.2, 1970.0 and 1997.3 respectively reaching $7.1 \%, 9.6 \%$ and $6.2 \%$ of the Earth's surface.

(ii) For the $\ddot{Y}$ component, four maxima appear in 1969.9, $1977.4,1985.2$ and 1997.5 respectively reaching $8.6 \%$, $10.0 \%, 5.1 \%$ and $5.8 \%$.

(iii) The $\ddot{Z}$ component analysis seems to be more sensitive to the rapid changes of the zero isoline, indeed, eight epochs with values of $S(t)$ greater than $5 \%$ can be noted: 1967.3, 1970.2, 1972.8, 1980.6, 1984.6, 1987.9, 1989.5 and 1997.3.

Because they are calculated with a 0.1 year interval, these percentages may appear low compares with, for example, a six-month analysis. The dates obtained are close to those already admitted as epochs for geomagnetic jerks, around 1968 (from 1967 to 1972), 1978 (from 1977 to 1981), 1990 (from 1988 to 1991) and 1999 (from 1997 to 2000). However, the $S(t)$ function does not present only one maximum for each of these events, and, another event around 1985 appears clearly in the $\ddot{Y}$ and $\ddot{Z}$ curves. 

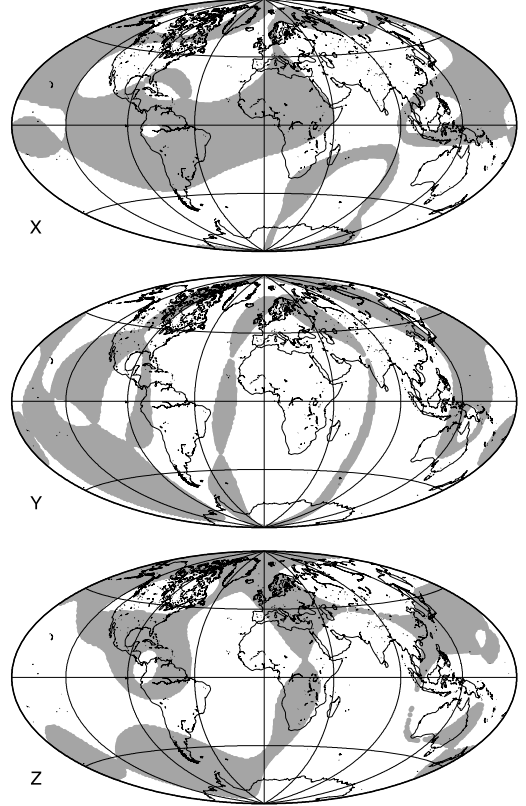

Fig. 5. Same caption as Fig. 3 but for one one-year period centred onto: 1985.0.

Each of the Figs. 3 to 6 corresponds to an event and shows locations where the zero isoline of $\ddot{E}$ swept the Earth's surface, so where the geomagnetic jerk appears. A thin (respectively, a thick) band occurs where a zero isoline moves a little (respectively, a lot). We specify also that the number of panels is different from one epoch to another one, as we represent maps corresponding to the maxima $S(t) \geq$ $5 \%$, thus, geomagnetic jerks are no longer considered either strictly abrupt or truly worldwide, but may instead take months to sweep across an appreciable fraction of Earth's surface.

Figure 3 shows maps obtained for three periods corresponding of the three $S(t)$ maxima representative of the 1968 event (e.g. 1966.7-1967.7, 1969.5-1970.5 and 1972.2-1973.2). During the first one-year period, different small-size regions are "swept" by the zero isoline. The event is observed in the Southern hemisphere in the northern component $(\ddot{X})$ and less defined in the two others components. For the second one-year period, the three components present a clear peak in $S(t)$. The areas covered by zero isolines are mainly concentrated above the Northern hemisphere, and especially above Europe and Asia. For the last period, the larger covered surfaces are the South Atlantic and the South Eastern Pacific, and this, mainly in the $\ddot{Z}$ component.

Figure 4 shows, from left to right, maps obtained for two periods corresponding to the two $S(t)$ maxima representative of the 1978 event (e.g. 1977.0-1978.0 and 1980.01981.0). Both panels show that this event is not clearly present in the $\ddot{X}$ component. For the first one-year period, in the $\ddot{X}$ and $\ddot{Z}$ maps, large areas are covered by the zero isoline, mainly in the Southern hemisphere especially in the South Indian Ocean and South Atlantic. In the second oneyear period, the map corresponding to $\ddot{Z}$ is the one showing the largest surface covered by the geomagnetic jerk especially above Europe.
On Fig. 5, the maps show large areas covered by zeroisoline of $\ddot{X}, \ddot{Y}$ and $\ddot{Z}$ that traduce a fast movement of the zero isolines of each component during the period 1984.51985.5. The largest compact area corresponding to $S(t) \geq$ $5 \%$ is situated in the Pacific hemisphere. Even if no geomagnetic jerk has been noted until now for this epoch, it becomes clear that we can expect to find such an event in observatory data located in this region.

Figure 6 shows, from left to right, maps obtained for two periods corresponding to the two $S(t)$ maxima representative of the 1990 event (1987.5-1988.5 and 1989.0-1990.0). The maps obtained for the first one-year period clearly show a localised event above the European/African region for all three secular acceleration components. For the second panel, the event seems to be more clearly expressed in $\ddot{Y}$. However, the $S(t)$ maxima is given by $\ddot{Z}$ and, this can be explained by a large number of narrow surfaces covered by zero isolines. This is reflected by the wide temporal window shown by the considered $S(t)$ peak.

This rough analysis allows us to obtain clues about the morphology of the spatial extent of geomagnetic jerks at the Earth's surface. Because we are studying the temporal evolution of the magnetic field by means of a model, it is difficult to avoid the influence of the temporal basis functions used in the CM4 model. However, our analysis is able to show that: (i) the geomagnetic jerks detected in the CM4 model do not appear to be worldwide; (ii) the area covered by an event is more or less extended in space but also in time; (iii) the method applied reveals an important event around 1985 localised in the Pacific, which was not previously recognized.

Several questions arise concerning the above conclusions: Is the 1985 event a real geomagnetic jerk? Are there some signatures of these events at the top of the core? Do the core flows present localised features at the same locations around the same epochs? If not, is there a parameter of the core flows that might be correlated with the three already-known geomagnetic jerks and the event of 1985? In the following, we try to answer these questions by starting with flow motions at the top of the core.

\section{Core Surface Flows}

A spectral method (e.g. Gire and Le Mouël, 1990) is used to compute core surface flows. This method relies on the magnetic induction equation, written at the top of the core, in which the magnetic diffusion is neglected according to the frozen-flux approximation (Roberts and Scott, 1965) :

$$
\frac{\partial B_{r}}{\partial t}=-\nabla_{\mathrm{H}} \cdot\left(\boldsymbol{u} B_{r}\right)
$$

$B_{r}$ being the radial component of the magnetic field, $\boldsymbol{u}$ the fluid velocity, assumed horizontal, and $\nabla_{\mathrm{H}}=\boldsymbol{\nabla}-\partial / \partial r$. To reduce the non-uniqueness of Eq. (1) the flow is assumed to be tangentially geostrophic (Hills, 1979; Le Mouël, 1984; Backus and Le Mouël, 1986, 1987; see also the derivation of the geostrophic vertical vorticity equations in Voorhies, 1991-Appendix B). The radial magnetic field $B_{r}$ and its secular variation $\partial B_{r} / \partial t$ observed at the Earth's surface are downward continued to the surface of the core (e.g. Blakely, 1995). The velocity field $\boldsymbol{u}$ is estimated by inverting, with 

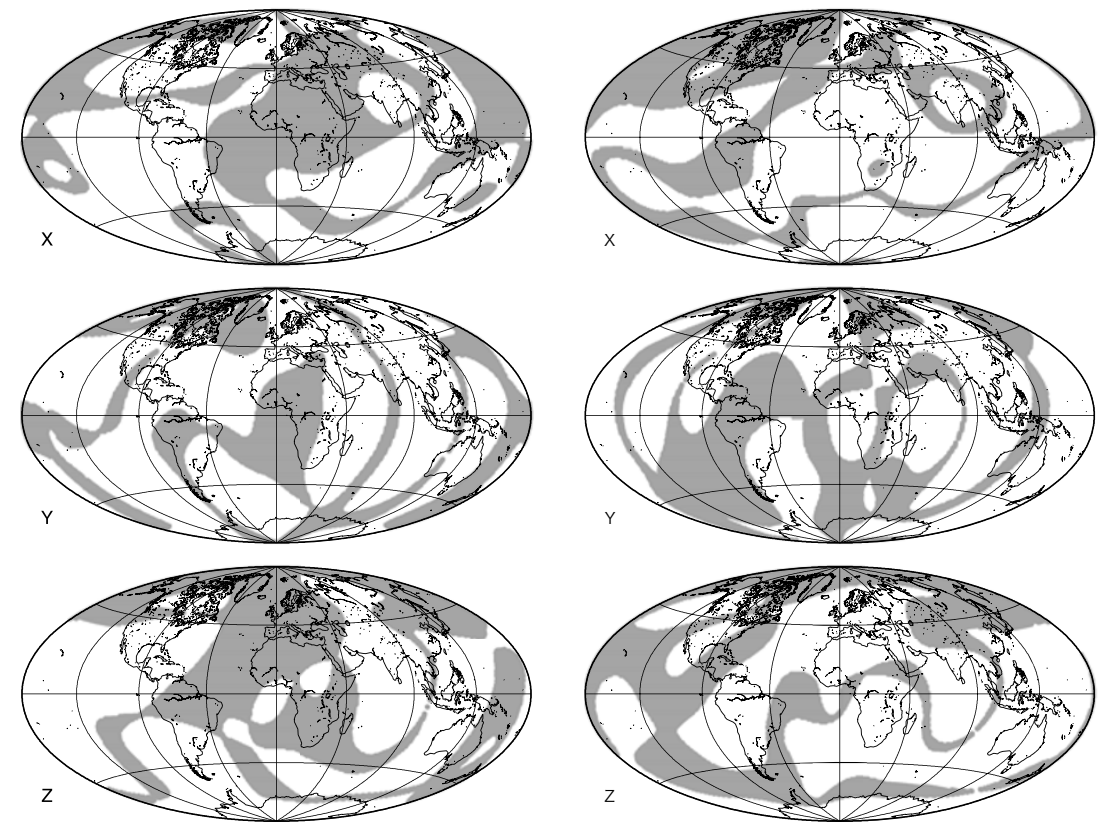

Fig. 6. Same caption as Fig. 3 but for two one-year periods centred onto: 1988.0 (left) and 1989.5 (right).

a Bayesian inverse method (e.g. Menke, 1984), a matrixform of Eq. (1) obtained by using spherical harmonic expansions of the magnetic and velocity fields. The obtained flow model represents the flow fitting the observed secular variation as well as possible-given a priori observational uncertainties-, with as little small scale structure as possible (a detailed description of the method used is given by Pais and Hulot, 2000). Two parameters have to be determined a priori: the covariance matrix, that specifies the observational uncertainties associated to the secular variation, and the damping parameter, that states the ratio between finding a flow that reasonably fits the secular variation, given the observational uncertainties, and finding a flow in which small-scales are not too energetic (see Eymin and Hulot, 2005, for a detailed discussion of this last point). In this study we chose to keep the parameters used by Pais and Hulot (2000) because of their good adaptation for both ground and satellite data (see Eymin (2004) and Eymin and Hulot (2005), for more details).

Core surface flows are estimated each 0.1 year between 1962.5 and 2000 (the first and last 2.5 years of the period covered by the CM4 model are not used to avoid artifacts linked to possible edge effects). The core flow estimation does not constrain the flow to match secular acceleration (or higher time derivatives) during such brief intervals. Each flow model is computed independently from the others and no special condition is imposed at jerk times.

The tangential geostrophy assumption used to compute these core surface flows implies that the fluid acceleration has been neglected in the momentum transport equation. Even if this assumption is not a very strong one (see Voorhies, 1995, for example), it has been shown that a purely steady flow cannot account for the evolution of the magnetic field over time span of the order of 10 year (Jackson, 1997). We thus compute here a piecewise constant flow. It is assumed to be constant between two time steps but is allowed to vary at each time step.

The obtained velocity fields at the core surface are in good agreement with previous computations. The flow pattern evolves slowly and no special modification of the flow is visible at jerk times. Figure 7 shows the velocity obtained for the 1970 and 1990 epochs. The three main structures appearing in most of core surface flow computations (Jault, 1990; Eymin, 2004) also exist in our models. They indeed exhibit the Atlantic westward current, as well as the two anticyclones located beneath Northern America and the Southern Indian Ocean. As these structures are visible in most of the recent flow models computed with different methods and under different assumptions (Holme and Wahler, 2001; Amit and Olson, 2004; Pais et al., 2004; Eymin and Hulot, 2005), they can be considered as the most reliable at the core surface.

Each individual flow model, corresponding to a given date, reproduces the instantaneous secular variation. The fact that each flow model is "independent" (independence limited by the fact that fitted data are somehow serially correlated through the cubic B-splines of the used magnetic field model) from the others does not insure that the time series obtained by juxtaposing the instantaneous flow models correctly explains the jerk signals (see Davis and Whaler, 1997). Nevertheless, Fig. 8 shows that the flow time series globally fits the jerk characteristics. Indeed, the secular variation predicted by the flow models effectively contains jerks, seen as a change of the slope of the eastern component of the secular variation. These events appear smoother from core surface flows than in observatory data. This happens because the magnetic field model that is itself smoother than observatory data.

\section{Pressure Maxima and Jerks}

As already mentioned, the global pattern of the velocity field exhibits no special feature during jerk events. How- 

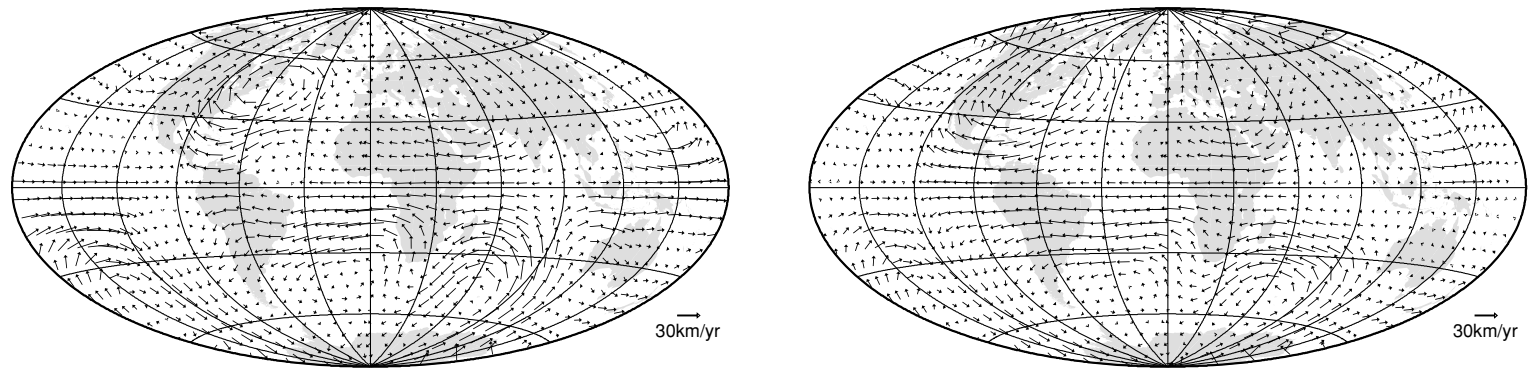

Fig. 7. Core surface flows in 1970 (left) and 1990 (right) obtained from the CM4 model. Arrow sizes are proportional to the fluid velocity.
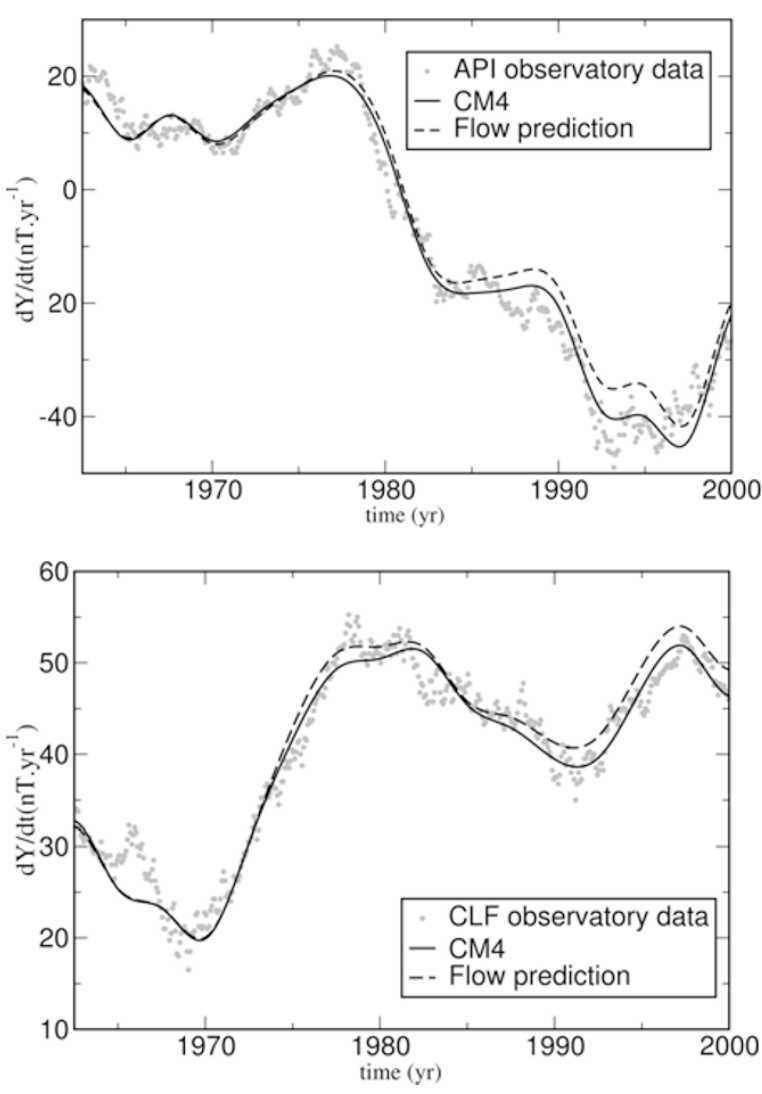

Fig. 8. $\quad \dot{Y}$ secular variation components predicted by the core surface flow model (dashed line), by the model CM4 (solid line) and obtained directly from observatory monthly means (grey dots). The comparison is displayed for API (Apia, Western Samoa) observatory (top) and CLF (Chambon la Forêt, France) observatory (bottom).

ever, we observe an intriguing behaviour considering the mean pressure amplitude $<|p|>$, defined as:

$$
<|p|>=\frac{1}{S} \int_{S}|p| d S
$$

$S$ being the surface of the core and $p$ the dynamical pressure associated with the flow. The pressure and velocity fields are linked by $\boldsymbol{\nabla}_{\mathrm{H}} p=-2 \rho(\boldsymbol{\Omega} \wedge \boldsymbol{u}), \boldsymbol{\Omega}$ being the Earth's rotation, because of the tangentially geostrophic assumption (Gire, 1985). Figure 9 shows that each maximum of the mean pressure amplitude happens at the time of, or just before, the already known occurrence of the geomagnetic jerks, except the maximum in 1985.

To understand this maximum, we investigate whether a jerk, not yet identified in observatory time series, happened

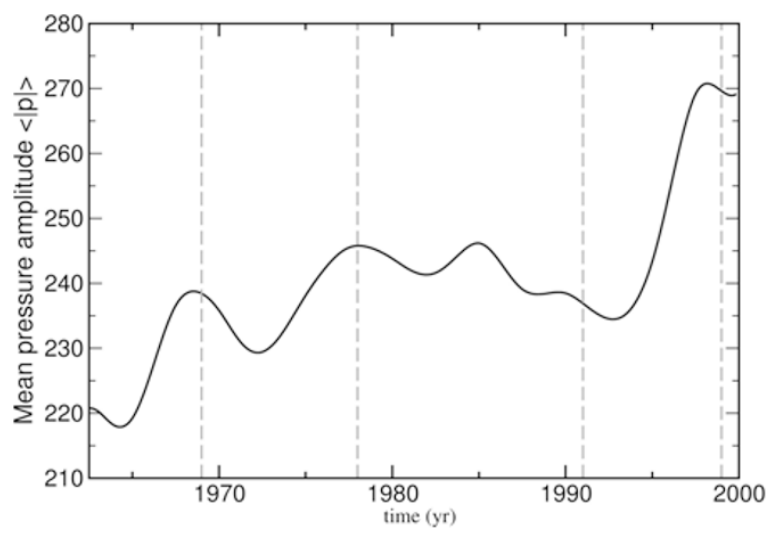

Fig. 9. Mean amplitude of the dynamical pressure at the core surface (in Pa). Vertical lines indicate the occurrence epochs of the widely recognised geomagnetic jerks: 1969, 1978, 1991 and 1999. A fifth local maximum in global mean absolute pressure occurs at the epoch 1985 jerk identified in this study.

around this date. Examination of observatory series confirm the existence of a jerk signal around 1985. Figure 10 shows the evidence in four of them, particularly in the $\dot{Z}$ component. The characteristic V-shape of a geomagnetic jerk in the secular variation is, however, more clear in the southern and western observatories (API, GUA, and PPT) than in the northern one (HON). The fact that very few observatories are run in the Southern Pacific, where this event is mainly visible, could explain why it has not yet be noticed.

This correlation between geomagnetic jerks and pressure maxima at the core surface can also be seen on a longer time span. Computing core surface flow for the period 1900-1990, using the gufm1 model (Jackson et al., 2000), confirms this striking correlation. Pressure maxima are indeed found for each known jerk and at no other time. However, the 1985 jerk is not clearly observed when the gufm 1 model is used, which we attribute to the rapidity of the event.

\section{Discussion and Conclusions}

A global characterisation of geomagnetic jerks is difficult to achieve when only data provided by observatories are available: they are very unevenly distributed in space, being concentrated over the continents of the northern hemisphere while being sparser over the oceans and over the southern hemisphere. Nevertheless, using these very valuable and long series of data, and applying different mathematical tools, some questions have been already addressed about 

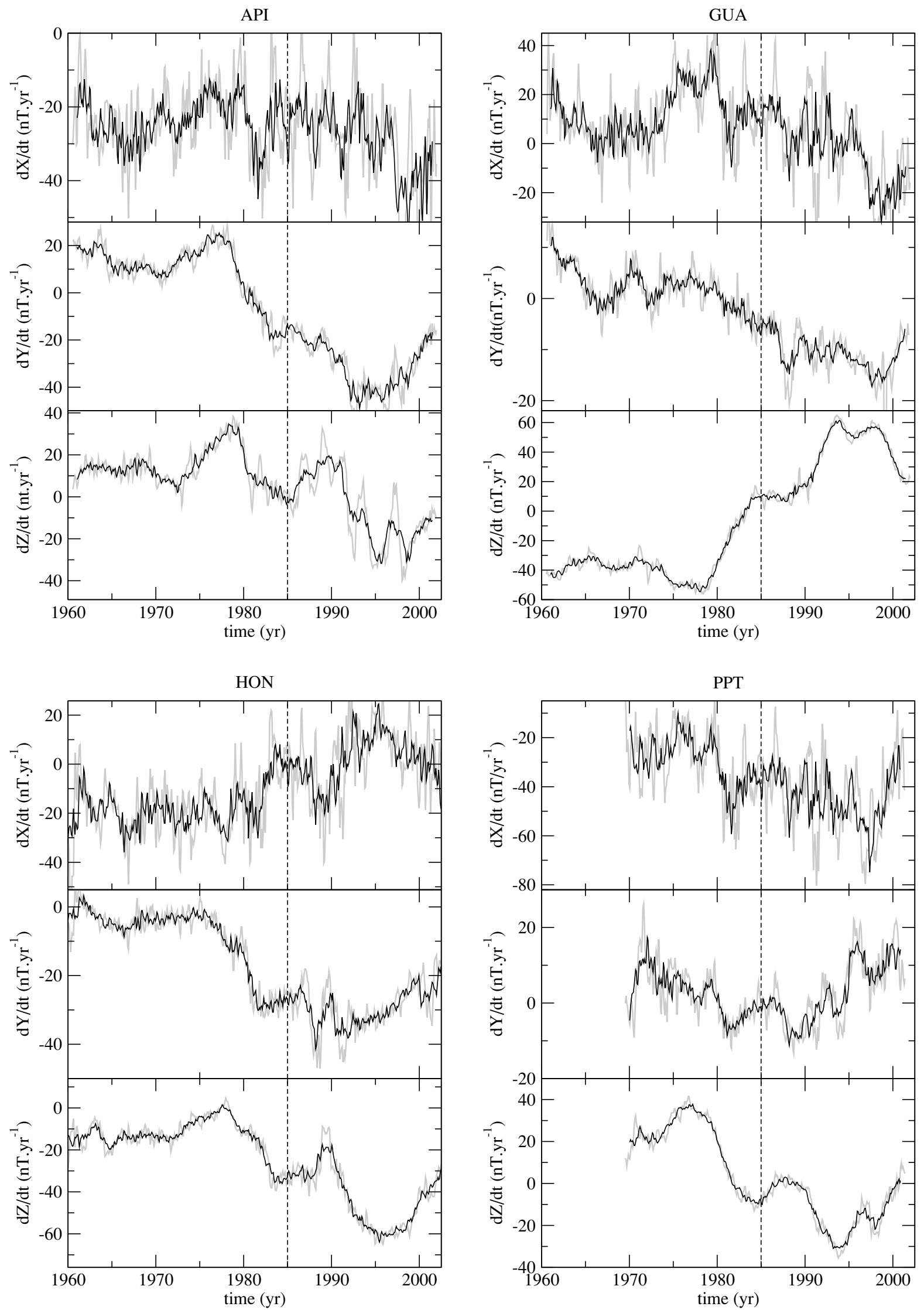

Fig. 10. $\dot{X}, \dot{Y}$ and $\dot{Z}$ secular variation components calculated from observatory monthly means: API (Apia, Western Samoa), GUA (Guam, Marianna Islands), HON (Honolulu, Hawaii) and PPT (Pamatai, French Polynesia). Short periods have been filtered out by a 12-months (grey line) or 24-months (black line) running averages. The 1985 geomagnetic jerk is indicated by a vertical dashed line. 
the spatial and temporal occurrence of geomagnetic jerks (Alldredge, 1984, 1985; McLeod, 1985; Alexandrescu et al., 1996; De Michelis et al., 1998; Mandea et al., 2000; Chambodut and Mandea, 2005).

In this paper we show that the comprehensive model of Sabaka et al. $(2002,2004)$ is a valuable and useful tool for detecting geomagnetic jerks in synthetic series obtained from it. Here, we focus on the spatial occurrence of geomagnetic jerks by exploiting the information contained in the second derivative of each of the magnetic field component. The 2.5-year knot spacing, used in the initial modeling approach, obviously appears in the results. Nevertheless, this drawback seems to be partially circumvented. We find that a sudden event in 1985, localised mainly in the Pacific hemisphere, corresponds to a geomagnetic jerk (Chambodut, 2004; Eymin, 2004). Our statement that geomagnetic jerks might not be worldwide in occurrence, has been recently underlined by Olsen and Mandea (2007), with the evidence of a regional 2003 geomagnetic jerk, directly detected in the CHAMP satellite data.

In order to validate the 1985 event, we compute core flows from the CM4 model and search for signatures linked to geomagnetic jerks. The maxima observed in the mean dynamical pressure amplitude, computed over the 42 years covered by the CM4 model, are at dates corresponding to the three already-known geomagnetic impulses and the one of 1985. This link between pressure maxima and jerk epochs may appear, at first sight, similar to modifications of the flow energy associated to geomagnetic jerks (Hulot et al., 1993). Nevertheless, this is not exactly the case. Even if the pressure and velocity fields are related by the tangential geostrophic assumption, pressure and energy maxima do not always appear simultaneously in our flow time series. As a result, the signature of a geomagnetic jerk in the flow energy is less clear than in the dynamical pressure: each energy maximum does not correspond to a jerk, and each jerk does not correspond to an energy maximum or to a change of the energy slope (Hulot et al., 1993). The existence of a pressure maximum during a geomagnetic jerk is not contradictory with the results recently published by Bloxham et al. (2002). Pressure maxima indeed result in a smoothly time-varying flow that does not exhibit other special feature at jerk times. The flow models we obtain are more complex than those by Bloxham et al. (2002) but they do explain a more complex signal.

Understanding the physical meaning of the localised occurrence of geomagnetic jerks at the Earth's surface and of the observed pressure maxima is an open and interesting question. The question of the nature of the link between jerks and maxima of the mean absolute pressure at the surface of the core is beyond the scope of this study. One can however note that a maximum of the mean absolute pressure implies a peak in the absolute horizontal pressure gradient. This indicates a peak in the flow component perpendicular to the rotation axis that will have profound implication for core dynamics. There are several possible explanations for the fact that geomagnetic jerks might not be worldwide in occurrence:

(i) The abrupt magnetic changes generated at the core- mantle boundary diffuse through the mantle, which may act as a "filter" that would delay locally the arrival of the geomagnetic jerk at the Earth's surface. The mantle conductivity models are far from being well constrained. If some upper boundary limits for the lower mantle conductivity are proposed (Achache et al., 1980; Backus, 1983; Mandea Alexandrescu et al., 1999), the lateral conductivity variations are still too difficult to be determined (Nagao et al., 2003).

(ii) The morphology of the magnetic field itself may be rather more complex than what was expected (Voorhies, 2004a, 2004b). In particular, it is possible that the importance of narrow scale flows, their organisation, may be underestimated. That would explain why some events appear well localised in space on short periods of time, while some others, are less clearly defined.

(iii) The correlation of pressure maxima and epochs of geomagnetic jerks seems to be to point either to a special event in the bulk core dynamics or to a very short time scale fluctuations of the geodynamo.

Further studies will help in elucidating the origin of geomagnetic jerks: what are the processes required to cause secular variation to vary so abruptly at some given epochs, or alternatively, why does secular variation evolve "linearly" between these events?

Acknowledgments. All maps were plotted using the GMT software (Wessel and Smith, 1991). Coerte Voorhies is thanked for providing an extremely thorough and useful review. We also would like to thank an anonymous referee for comments which improved the manuscript.

\section{References}

Achache, J., V. Courtillot, J. Ducruix, and J.-L. Le Mouël, The late 1960's secular variation impulse: Further constraints on deep mantle conductivity, Phys. Earth Planet. Inter., 23, 72-75, 1980.

Alexandrescu, M., D. Gibert, G. Hulot, J.-L. Le Mouël, and G. Saracco, Detection of geomagnetic jerks using wavelet analysis, J. Geophys. Res., 100(B7), 12557-12572, 1995.

Alexandrescu, M., D. Gibert, G. Hulot, J.-L. Le Mouël, and G. Saracco, Worldwide wavelet analysis of geomagnetic jerks, J. Geophys. Res., 101, 21975-21994, 1996.

Alldredge, L. R., A discussion of impulses and jerks in the geomagnetic Field, J. Geophys. Res., 89, 4403-4412, 1984.

Alldredge, L. R., More on the alleged 1970 geomagnetic jerk, Phys. Earth Planet. Inter., 39, 255-264, 1985.

Amit, H. and P. Olson, Helical core flow from geomagnetic secular variation, Phys. Earth Planet. Inter., 147, 1-25, 2004.

Backus, G. E., Application of mantle filter theory to the magnetic jerk of 1969, Geophys. J. R. Astron. Soc., 74, 713-746, 1983.

Backus, G. E. and J.-L. Le Mouël, The region on the core-mantle boundary where a geostrophic velocity field can be determined from frozen flux magnetic data, Geophys. J. R. Astr. Soc., 85, 617-628, 1986.

Backus, G. E. and J.-L. Le Mouël, The region on the core-mantle boundary where a geostrophic velocity field can be determined from frozen-flux magnetic data-Addendum, Geophys. J. R. Astron. Soc, 88, 321-322, 1987.

Backus, G. E., R. H. Estes, D. Chinn, and R. A. Langel, Comparing the jerk with other global models of the geomagnetic Field from 1960 to 1978, J. Geophys. Res., 92, 3615-3622, 1987.

Bellanger, E., J.-L. Le Mouël, M. Mandea, and S. Labrosse, Chandler Wobble and geomagnetic Jerks, Phys. Earth Planet. Inter., 124,95-103, 2001.

Blakely, R., Potential theory in gravity and magnetic applications, Cambridge University Press, 1995.

Bloxham, J., On the consequences of strong stable stratification at the top 
of the Earth's outer core, Geophys. Res. Lett., 17, 2081-2084, 1990.

Bloxham, J., S. Zatman, and M. Dumberry, The origin of geomagnetic jerks, Nature, 420, 65-68, 2002.

Chambodut, A., Le champ magnétique terrestre : structures spatiales et variations temporelles vues par les Ondelettes, Ph.D. thesis, Institut de Physique du Globe de Paris, 2004.

Chambodut, A. and M. Mandea, Evidence for geomagnetic jerks in comprehensive models, Earth Planets Space, 57, 139-149, 2005.

Courtillot, V., J. Ducruix, and J.-L. Le Mouël, Sur une accélération récente de la variation séculaire du champ magnétique terrestre, C. R. Hebd. Séances Acad. Sci., ser. D, 287, 1095-1098, 1978.

Courtillot, V. and J.-L. Le Mouël, Comment on "discussion of impulses and jerks in the geomagnetic field" by L. R. Alldredge, J. Geophys. Res., 90, 6897-6898, 1985.

Courtillot, V., J.-L. Le Mouël, and J. Ducruix, On Backus' mantle filter theory and the 1969 geomagnetic impulse, Geophys. J. Roy. Astron. Soc., 78, 619-624, 1984.

Davis, R. and K. Whaler, Velocity fields at the Earth's core surface from 1900 to 1980 using the frozen-flux approximation and steady velocity assumption, Geophys. Astrophys. Fluid Dynamics, 67, 241-258, 1993.

Davis, R. and K. Whaler, The 1969 geomagnetic impulse and spin-up of the Earth's liquid core, Phys. Earth Planet. Inter., 103, 181-194, 1997.

De Michelis, P., L. Cafarella, and A. Meloni, Worldwide character of the 1991 geomagnetic jerk, Geophys. Res. Lett., 25, 377-380, 1998

Eymin, C., Etude des mouvements à la surface du noyau terrestre : du 17ème au 21ème siècle, Ph.D. thesis, Université Paris VI, 2004.

Eymin, C. and G. Hulot, On core surface flows inferred from satellite magnetic data, Phys. Earth Planet. Inter., 152, 200-220, 2005.

Gavoret, J., D. Gibert, M. Menvielle, and J.-L. Le Mouël, Long-term variations of the external and internal components of the Earth's magnetic field, J. Geophys. Res., 91, 4787-4796, 1986.

Gire, C., Sur la variation séculaire du champ magnétique terrestre et les mouvements des couches externes du noyau fluide, Ph.D. thesis, University of Paris 7, 1985.

Gire, C., J.-L. Le Mouël, and T. Madden, Motions of the core surface derived by SV data, Geophys. J. R. Astron. Soc., 84, 1-29, 1986.

Gire, C. and J.-L. Le Mouël, Tangentially geostrophic flow at core-mantle boundary compatible with the observed geomagnetic secular variation: The large-scale component of the flow, Phys. Earth Planet. Inter., 59. 259-287, 1990.

Golovkov, V. P., T. I. Zvereva, and A. O. Simonyan, Common features and differences between jerks of 1947, 1958 and 1969, Geophys. Astrophys. Fluid Dyn., 49, 81-96, 1989.

Gubbins, D., Finding core motions from magnetic observations, Phil. Trans. R. Soc. Lond., A306, 247-254, 1982.

Gubbins, D., Geomagnetic field analysis - II. Secular variation consistent with a perfectly conducting core, Geophys. J. Roy. Astron. Soc., 77, 753766, 1984.

Hills, R. G., Convection in the Earth's mantle due to viscous shear at the core-mantle interface and due to large-scale buoyancy, Ph.D. thesis, New Mexico State University, Las Cruces, 1979.

Holme, R. and K. Whaler, Steady core flow in an azimuthally drifting reference frame, Geophys. J. Int., 145, 560-569, 2001.

Hulot, G., M. Le Huy, and J.-L. Le Mouël, Secousses (jerks) de la variation séculaire et mouvements dans le noyau terrestre, C. R. Acad. Sci., 317, 333-341, 1993.

Jackson, A., Time-dependency of tangentially geostrophic core surface motions, Phys. Earth Planet. Inter., 103, 293-311, 1997.

Jackson, A., A. Jonkers, and M. Walker, Four centuries of geomagnetic secular variation from historical records, Phil. Trans. R. Soc. Lond., 358, 957-990, 2000.

Jault, D., Variation séculaire du champ géomagnétique et fluctuations de la longueur du jour, Ph.D. thesis, Université Paris VII, 1990

Le Huy, M., M. Mandea, G. Hulot, and J. Le Mouël, On the characteristics of successive geomagnetic jerks, Earth Planets Space, 50, 723-732, 1998.

Le Huy, M., M. Mandea, J.-L. Le Mouël, and A. Pais, Time evolution of the fluid flow at the top of the core-Geomagnetic jerks, Earth Planets Space, 52, 163-173, 2000

Le Mouël, J.-L., Outer-core geostrophic flow and secular variation of Earth's geomagnetic field, Nature, 311, 734-735, 1984.

Le Mouël, J.-L., C. Gire, and T. Madden, Motions at the core surface in the geostrophic approximation, Phys. Earth planet. Inter., 39, 270-287,
1985

Macmillan, S., A geomagnetic jerk for the early 1990's, Earth Planet. Sci. Lett., 137, 189-192, 1996.

Malin, S. and B. Hodder, Was the 1970 geomagnetic jerk of internal or external origin?, Nature, 296, 726-728, 1982.

Malin, S., B. Hodder, and D. Barraclough, Geomagnetic variation: a jerk in 1970, in 75th Anniversary Volume of Ebro Observatory, edited by $\mathrm{J}$. O. Cardus, 239-256, Ebro Obs., Tarragona, Spain, 1983.

Mandea, M., E. Bellanger, and J.-L. Le Mouël, A geomagnetic jerk for the end of the 20th century?, Earth Planets Space Letters, 183, 369-373, 2000

Mandea Alexandrescu, M., D. Gibert, J.-L. Le Mouël, G. Hulot, and G Saracco, An estimate of average lower mantle conductivity by wavelet analysis of geomagnetic jerks, J. Geophys. Res., 104, 17735-17745, 1999.

McLeod, M., On the geomagnetic jerk of 1969, J. Geophys. Res., 90 (B6), 0148-0227, 1985.

Menke, W., Geophysical data analysis: Discrete inverse theory, Academic Press, 1984

Nagao, H., T. Iyemori, T. Higuchi, S. Nakano, and T. Araki, Local time features of geomagnetic jerks, Earth Planets Space, 54, 119-131, 2002. Nagao, H., T. Iyemori, T. Higuchi, and T. Araki, Lower mantle conductivity anomalies estimated from geomagnetic jerks, J. Geophys. Res., 108 2254, 2003

Olsen, N. and M. Mandea, Investigation of a secular variation impulse using satellite data: The 2003 geomagnetic jerk, Earth Planet. Sci. Lett., 255, 94-105, doi:10.1016/j.epsl.2006.12.008, 2007.

Pais, A. and G. Hulot, Length of day decade variations, torsional oscillations and inner core superrotation: Evidence from recovered core surface zonal flows, Phys. Earth Planet. Inter., 118, 291-316, 2000.

Pais, M. A., O. Oliveira, and F. Nogueira, Non-uniqueness of inverted CMB flows and deviations from tangential geostrophy, J. Geophys. Res, 109, B08105-1-B08105-20, 2004.

Roberts, P. H. and S. Scott, On the analysis of the secular variation: 1. A hydromagnetic constraint: theory, J. Geomagn. Geoelectr., 17, 137-151, 1965.

Sabaka, T., N. Olsen, and R. A. Langel, A comprehensive model of the quiet-time, near-Earth magnetic field: phase 3, Geophys. J. Int., 151 32-68, 2002.

Sabaka, T., N. Olsen, and M. Purucker, Extending Comprehensive Models of the Earth's Magnetic Field with Ørsted and CHAMP data, Geophys J. Int., 159, 521-547, doi: 10.1111/j.1365246X.2004.02,421.x, 2004.

Voorhies, C. V., Magnetic Location of Earth's Core-Mantle Boundary and Estimates of the Adjacent Fluid Motion, Ph.D. thesis, University of Colorado, 1984.

Voorhies, C. V., Steady flows at the top of Earth's core derived from geomagnetic field models, J. Geophys. Res., 91, 12 444-12 466, 1986.

Voorhies, C. V., Coupling an Inviscid Core to an Electrically Insulating Mantle, J. Geomagn. Geoelectr., 43, 131-156, 1991.

Voorhies, C. V., Time-varying fluid-flow a the top of Earth's core derived from definitive geomagnetic reference field models, J. Geophys. Res., 100, 10,029-10,039, 1995.

Voorhies, C. V., Narrow-scale flow and a weak field by the top of Earth's core: Evidence from Ørsted, Magsat, and secular variation, J. Geophys. Res., 109, B03106, doi:10.1029/2003JB002833, 2004a.

Voorhies, C. V., Correction to Narrow-scale flow and a weak field by the top of Earth's core: Evidence from rsted, Magsat, and secular variation, J. Geophys. Res., 109, B08103, doi:10.1029/2004JB003289, 2004 b.

Voorhies, C. V. and G. E. Backus, Steady flows at the top of the core from geomagnetic-field models the steady motions theorem, Geophys. Astrophys. Fluid Dyn., 32, 163-173, 1985.

Waddington, R., D. Gubbins, and D. Barber, Geomagnetic field analysis5. Determining steady core-surface flows directly from geomagnetic observations, Geophys. J. Int., 122, 326-350, 1995.

Wessel, P. and W. H. F. Smith, Free software helps map and display data Eos Trans. AGU, 72, 1991.

Whaler, K. A., Does the whole of the Earth's core convect?, Nature, 287, 528-530, 1980

A. Chambodut (e-mail: aude@eost.u-strasbg.fr), C. Eymin, and M. Mandea 